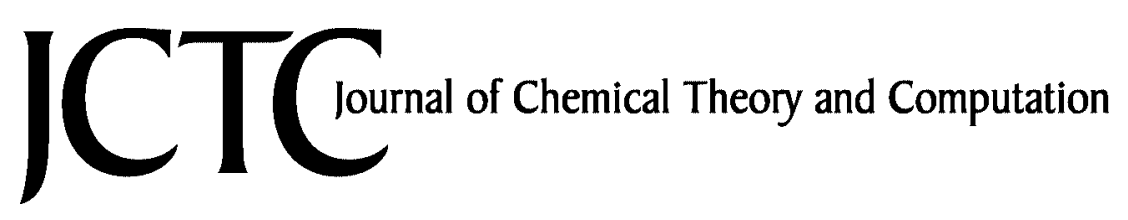

\title{
Structure Dependence of Hyperpolarizability in Octopolar Molecules
}

\author{
C. Cardoso, ${ }^{*}{ }^{\dagger}$ P. E. Abreu, ${ }^{\ddagger}$ and F. Nogueira ${ }^{\dagger}$ \\ CFC, Physics Department, Coimbra University, P-3004-516, Coimbra, Portugal, and \\ Chemistry Department, Coimbra University, P-3004-535, Coimbra, Portugal
}

Received October 31, 2008

\begin{abstract}
Recent Hyper Rayleigh Scattering measurements report a significant increase of second-order hyperpolarizability upon introduction of positive charges at the pyridyl end groups in trispyridyl octopolar chromophores. We calculated the geometries, linear response, and firstorder hyperpolarizabilities of a series of six trispyridyl molecules both in the neutral and protonated forms. The calculations were performed with $a b$ initio and semiempirical methods. The results are in good agreement with the experimental values and a correlation between the first hyperpolarizability and two structural properties, the $\mathrm{N}-\mathrm{C}$ bond elongation and the $\mathrm{C}-\mathrm{C}$ bond length alternation, $\langle\Delta r\rangle$ was established. To test these effects we computed the hyperpolarizability for several constrained geometries and confirmed the importance of planarity on the hyperpolarizability values. However the $\langle\Delta r\rangle$ values alone seem to have little influence both on the hyperpolarizability and on the gap values. Replacing the triple $\mathrm{C} \equiv \mathrm{C}$ bond by a double $\mathrm{C}=\mathrm{C}$ bond in the conjugation bridge has no significant effect due to the strong hyperpolarizability dependence on the pyridyl-benzene dihedral angle.
\end{abstract}

\section{Introduction}

Photonics is playing an ever-increasing role in today's technology by efficiently replacing electronics in electrooptic devices. In this context, researchers have been focusing on the search for materials with large optical nonlinearities and for their application in the fields of high speed data transmission, processing and storage. ${ }^{1-6}$ Organic molecules are promising candidates since their properties can be custom-tailored, and their dielectric constants and refractive indixes are much smaller than those of the most common inorganic molecules. A second-order Non-Linear Optical (NLO) chromophore typically contains a conjugated $\pi$-electron system, asymmetrically substituted by electron donor and acceptor groups, through which a charge transfer occurs. In such systems the dominant first hyperpolarizability component is along the direction of charge transfer.

In the early 1990s it was shown that, for a given conjugation bridge, there is an optimal combination of donor

* Corresponding author e-mail: cmcardoso@teor.fis.uc.pt.

$\dagger$ CFC, Physics Department.

Chemistry Department. and acceptor strengths or ground-state polarization to maximize the dipole moment-first hyperpolarizability product, $\mu \beta$. Beyond a certain point, increased donor-acceptor strength or further ground-state polarization attenuates $\mu \beta .^{7,8}$ Bond length alternation, i.e., the average difference in length between single and double bonds in the molecule, was also shown to be a relevant parameter in the optimization of the hyperpolarizability of molecules. ${ }^{9-11}$ Recently, the possibility of modulating NLO properties at the molecular level using molecules which respond to electrochemical or chemical inputs such as protons or metal cations has been explored. ${ }^{12-15}$ In chemosensitive systems of the donor- $\pi$-acceptor type, interaction with a cationic species alters the electron density of the terminal sites, resulting in a modulation of the internal charge transfer character of the dye molecule which leads to a change in the optical response. ${ }^{16-18}$ There have been several reports of modulation of the two-photon absorption and fluorescence properties of linear chromophores upon cation binding or protonation/deprotonation at the terminal donor substituent. ${ }^{19-22}$

The antiparallel alignment of the dipole moments of onedimensional chromophores leads the majority of $\pi$-conju- 


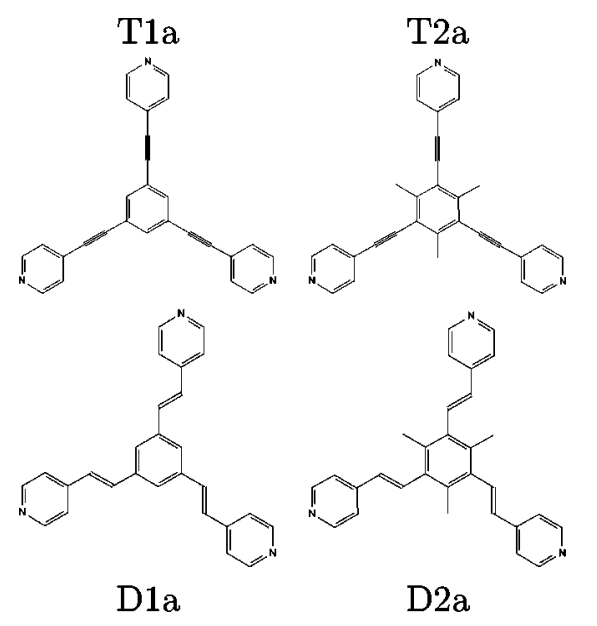

Figure 1. One example of each series: D1, D2, T1, and T2. The rest of the series corresponds to different locations of the nitrogen atom in the pyridyl rings, as shown in Figure 2.

gated organic molecules to crystallize in centrosymmetric space groups, having therefore null second-order bulk susceptibility. One of the solutions to achieve dipole minimization without losing the molecular hyperpolarizability is the use of noncentrosymmetric octopolar systems, since their symmetry ensures cancelation of the dipole moment. ${ }^{1,23-34}$ Despite the growing impact that octopolar systems are having in the field of nonlinear optics, few studies report on organic NLO octopoles with switchable second-order nonlinearity. ${ }^{35-38}$ Theoretical calculations on octopolar tris-acetylene benzenes suggest that the second-order hyperpolarizability $\beta$ could increase more than 1 order of magnitude upon introduction of positive charges at the pyridyl end groups. ${ }^{39-42}$ Asselberghs et al. report a strong increase of the charge transfer character and, consequently, of second-order hyperpolarizability upon introduction of positive charges at the terminal pyridyl groups of octopolar 1,3,5-trisalkynylbenzenes. ${ }^{37}$ In a work by Oliva et al. the origin of the first-order NLO response was attributed, for the neutral systems, to octopolar contributions, while the enhanced response after protonation was attributed to the involvement of dipolar excited states yielding dipolar contributions to the NLO outcome. ${ }^{38}$

In the present work we explore the relation between protonation, bond length alternation, and enhanced hyperpolarizability in octopolar chromophores. We performed calculations for several prototypical chromophores of neutral and protonated 1,3,5-trispyridylbenzene in order to determine the parameters that influence its hyperpolarizability. We studied the influence of the conjugation bridge by considering both a $\mathrm{C}-\mathrm{C} \equiv \mathrm{C}-\mathrm{C}$ bridge connecting the pyridyl rings to the central benzene ring (we refer to these systems as the $\mathbf{T}$ series) and a $\mathrm{C}-\mathrm{C}=\mathrm{C}-\mathrm{C}$ bridge (the $\mathrm{D}$ series, see Figure $1)$. We also compared the hyperpolarizability values for normal (T1 and D1 series) and methylated compounds (T2 and D2 series, Figure 1), both in the neutral and the protonated forms. Finally, we assessed the effect of the location of the pyridyl nitrogen (series T1a, T1b, etc. --see Figure 2).

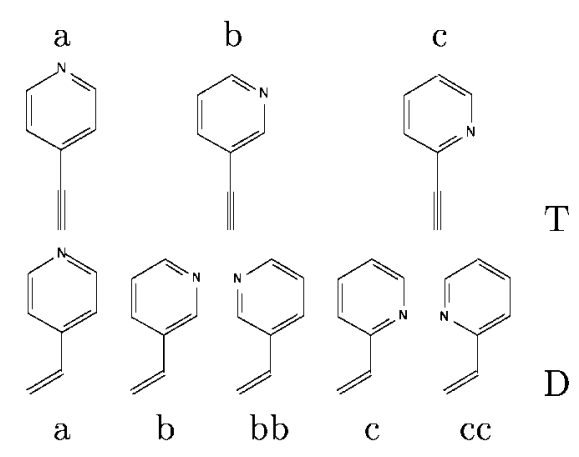

Figure 2. Nitrogen location in the pyridyl rings of the molecules under study.

Table 1. Calculated and Experimental First Hyperpolarizability Component $\beta_{x x x 0}$ for the T Series

\begin{tabular}{|c|c|c|c|c|c|}
\hline & B3LYP (au) & РM3 (au) & PM6 (au) & Exp. $^{37}(\mathrm{au})$ & $\mathrm{CPHF}^{38}(\mathrm{au})$ \\
\hline \multicolumn{6}{|c|}{ Neutral } \\
\hline $1 a$ & 804 & 796 & 753 & & \\
\hline $1 b$ & 229 & 240 & 525 & & \\
\hline $1 \mathrm{c}$ & 722 & 938 & 653 & 926 & 417 \\
\hline $2 a$ & 2446 & 1722 & 3262 & 1389 & 1346 \\
\hline $2 b$ & 1763 & 916 & 1920 & & \\
\hline $2 c$ & 2860 & 2002 & 3537 & 1041 & 1198 \\
\hline \multicolumn{6}{|c|}{$3+$} \\
\hline $1 a$ & 6415 & 6430 & 7405 & & \\
\hline $1 b$ & 3548 & 4701 & 4049 & & \\
\hline $1 c$ & 3438 & 4561 & 2350 & 2199 & 1844 \\
\hline $2 a$ & 9815 & 9848 & 8837 & 16895 & 5248 \\
\hline $2 b$ & 6231 & 6146 & 4070 & & \\
\hline $2 c$ & 5944 & 3302 & 2718 & 4397 & 3044 \\
\hline
\end{tabular}

\section{Computational Methods}

We obtained the ground-state geometries, hyperpolarizability, and optical properties of all these molecules within Density Functional Theory (DFT) and Time Dependent Density Functional Theory (TDDFT), using B3LYP for exchange and correlation. ${ }^{43,44}$ The molecular geometries were optimized with the GAMESS-US code ${ }^{45}$ using a $6-311 \mathrm{G}+(\mathrm{d}, \mathrm{p})^{46}$ basis set of wave functions. The static second-order hyperpolarizabilities were computed using the finite fields method within the same level of theory. The OCTOPUS $\operatorname{code}^{47,48}$ was used to compute the ground-state Electron Localization Function (ELF ${ }^{49}$ and the optical absorption spectra. The ground-state geometries and hyperpolarizability values were again computed using MOPAC2007, ${ }^{50}$ with the PM3 $3^{51-54}$ and $\mathrm{PM}^{55}$ Hamiltonians.

\section{Results and Discussion}

The discussion of our results will be done in four steps: i) comparison of our results for the second-order nonlinear polarizabilities with experimental (Hyper Rayleigh Scattering -- HRS) and other computational results; ii) comparison between the $\mathbf{T}$ and $\mathbf{D}$ series; iii) discussion of the computed molecular geometries and ELF, and their relation with the hyperpolarizability values; and iv) report on DFT energy levels and Kohn-Sham orbitals and linear response.

3.1. Nonlinear Optical Properties. In Table 1, we compare the static $\beta_{x x x 0}$ component computed for the $\mathbf{T}$ series within DFT/B3LYPPM3, and PM6 semiempirical methods 


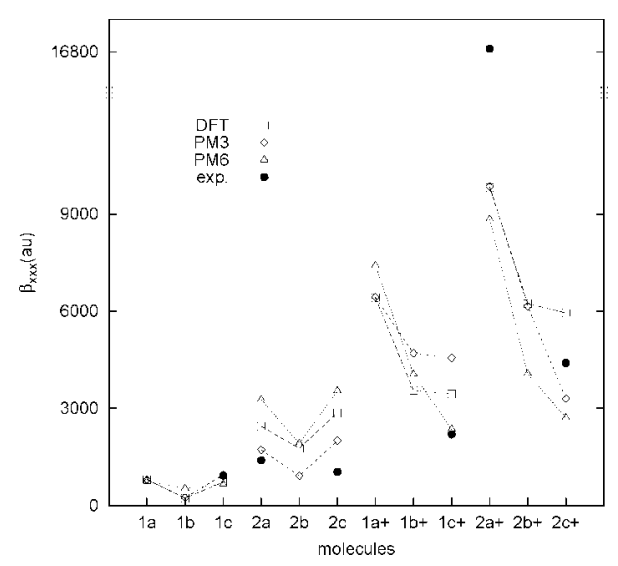

Figure 3. Comparison between calculated and experimen$\mathrm{tal}^{37}$ hyperpolarizability component $\beta_{x x \times 0}$ for the $\mathbf{T}$ series.

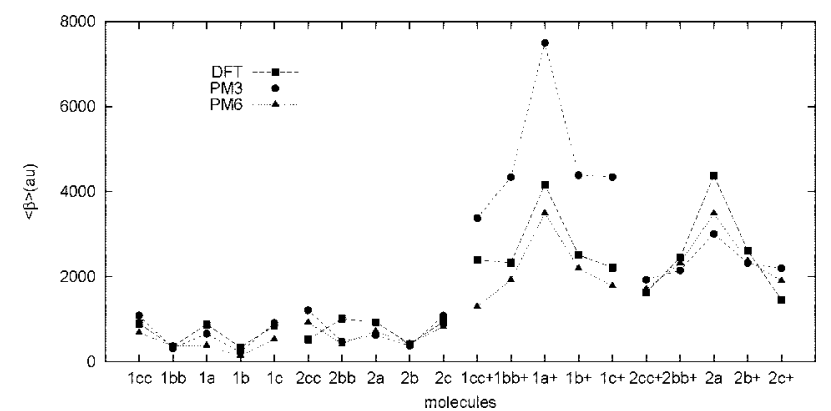

Figure 4. $\left\langle\beta_{H R}\right\rangle$ in atomic units, computed within DFT/B3LYP, PM3 and PM6 methods for the D series.

to HRS experimental values, obtained within the three level model. ${ }^{37}$ In order to make the comparison easier, the same results are plotted in Figure 3.

The present results, obtained both $a b$ initio and semiempirically, reproduce very well the trend of the experimental results. ${ }^{37}$ The increase of the computed hyperpolarizability with protonation and the larger hyperpolarizability of molecules belonging to the $\mathbf{2}$ series (compared with series $\mathbf{1}$ ) reflect the stronger donor character of the $\mathrm{CH}_{3}$ group and are in agreement with experiment. Within each of the neutral series the calculated hyperpolarizability values are very similar, whereas in the case of the protonated series the differences are larger: molecules a present hyperpolarizability values significantly larger than molecules $\mathbf{b}$ and c. This is particularly noticeable for the $\mathbf{T} \mathbf{2}$ series computed within the PM3 and PM6 methods. This is again in good agreement with the HRS results. The DFT/B3LYP results are also in good agreement with Coupled Perturbed Hartree-Fock (CPHF) results. ${ }^{38}$ The small differences can be attributed to the use of different basis sets and computation methods.

In Figure 6 we show also the intrinsic hyperpolarizability, $\beta_{\text {int }}$, as defined by Zhou et al. ${ }^{56}$ This property gives a scaleindependent intrinsic value, useful as a metric that takes into account the molecular size. In the case of the present series, and since the molecules have the same size and number of $\pi$ electrons, its value depends only on the energy gap. $\beta_{\text {int }}$ follows very closely the variation of the absolute $\beta$ values. The exceptions are the protonated T1c and T2c molecules, which show a small increase of $\beta_{\text {int }}$ with respect to $\mathbf{T} \mathbf{1 b}$ and $\mathbf{T} \mathbf{2 b}$, contrary to what happens to the absolute $\beta$.

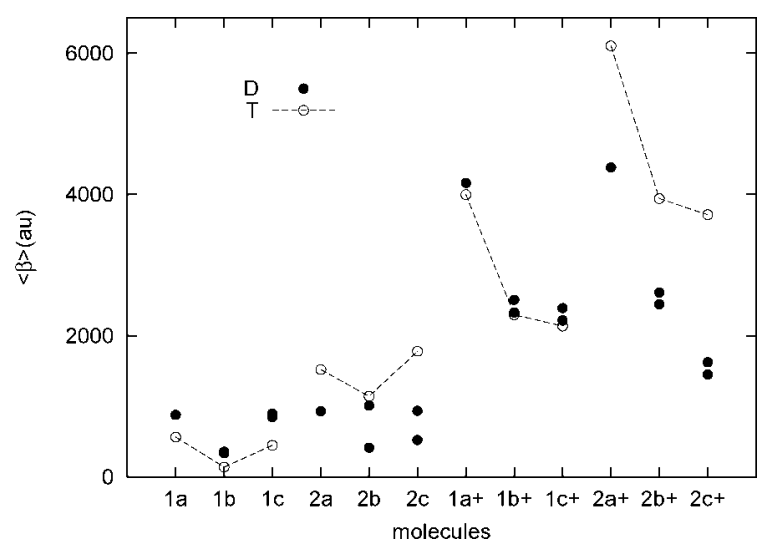

Figure 5. Comparison between the computed DFT/B3LYP $\left\langle\beta_{H R}\right\rangle$ values for the $\mathbf{T}$ and $\mathbf{D}$ molecules. In the plot, the $\mathbf{1} \mathbf{b}$, 1c, $\mathbf{2 b}$, and $\mathbf{2 c}$ symbols include also, for the $\mathbf{D}$ series, the values of $\mathbf{1 b b}, \mathbf{1 c c}, \mathbf{2 b b}$, and $\mathbf{2 c c}$.

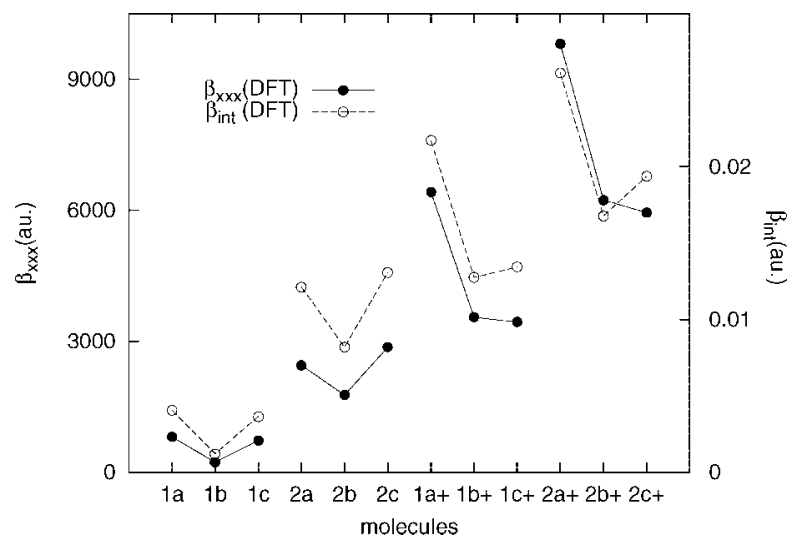

Figure 6. Comparison of the absolute hyperpolarizability values with the intrinsic hyperpolarizability.

Although the calculated values reproduce well the experimental trends, the absolute values show some discrepancies. The molecule with the largest hyperpolarizability presents also the largest deviation from the experimental results. Although DFT methods are expected to overestimate hyperpolarizability, as in fact happens for the other molecules, the measured value for $\mathbf{T} 2 \mathbf{a}^{3+}$ is 1.8 times larger than the computed $\beta_{x x x 0}$. The experimental value reported for this particular molecule is $16895 \pm 578$ au. The DFT/B3LYP values do not fall in this range. These differences may have several origins: the basis set, the exchange and correlation functional, and the neglect of solvent effects.

In order to test the basis set used in the $a b$ initio calculations we performed also some calculations with the Z3POL basis set, ${ }^{57,58}$ a basis set specially tailored for the calculation of electrical properties. The $\beta_{x x x 0}$ obtained with Z3POL for the neutral and protonated T2a molecules are 2352 and $9584 \mathrm{au}$, whereas the $6-311 \mathrm{G}+(\mathrm{d}, \mathrm{p})$ values are 2446 and $9815 \mathrm{au}$, respectively. The results are not significantly different, showing that $6-311 \mathrm{G}+(\mathrm{d}, \mathrm{p})$ is adequate for the task at hand.

Among the DFT exchange and correlation functionals used in the calculation of nonlinear optical properties, the hybrid methods, like the B3LYP used in the present study, represent a significant improvement on the pure DFT methods. ${ }^{59,60}$ Sałek et al. reports mean absolute errors of $9 \%$ for dynamic 
Table 2. $\left\langle\beta_{H R}\right\rangle$ in Atomic Units, Computed within DFT/ B3LYP, PM3, and PM6 Methods

\begin{tabular}{|c|c|c|c|c|c|c|}
\hline & \multicolumn{3}{|c|}{$\mathrm{D}$} & \multicolumn{3}{|c|}{$\mathrm{T}$} \\
\hline & B3LYP & PM3 & PM6 & B3LYP & PM3 & PM6 \\
\hline \multicolumn{7}{|c|}{ Neutral } \\
\hline $1 \mathrm{cc}$ & 898 & 1087 & 682 & - & - & - \\
\hline $1 \mathrm{bb}$ & 358 & 318 & 371 & - & - & - \\
\hline $1 a$ & 880 & 660 & 377 & 567 & 662 & 626 \\
\hline $1 b$ & 339 & 223 & 142 & 141 & 168 & 356 \\
\hline $1 \mathrm{c}$ & 847 & 918 & 526 & 449 & 622 & 504 \\
\hline $2 c c$ & 1011 & 1210 & 925 & - & - & - \\
\hline $2 b b$ & 523 & 477 & 421 & - & - & - \\
\hline $2 a$ & 932 & 626 & 715 & 1521 & 1178 & 2150 \\
\hline $2 b$ & 416 & 375 & 425 & 1142 & 692 & 1608 \\
\hline $2 c$ & 938 & 1082 & 822 & 1780 & 1370 & 2252 \\
\hline \multicolumn{7}{|l|}{$3+$} \\
\hline $1 \mathrm{cc}$ & 2393 & 3379 & 1293 & - & - & - \\
\hline $1 \mathrm{bb}$ & 2330 & 4345 & 1925 & - & - & - \\
\hline $1 a$ & 4162 & 7499 & 3495 & 3998 & 5392 & 4750 \\
\hline $1 b$ & 2510 & 4391 & 2197 & 2299 & 2902 & 2556 \\
\hline $1 \mathrm{c}$ & 2217 & 4345 & 1785 & 2139 & 2820 & 1692 \\
\hline $2 \mathrm{cc}$ & 1626 & 1933 & 1707 & - & - & - \\
\hline $2 b b$ & 2447 & 2151 & 2319 & - & - & - \\
\hline $2 a$ & 4384 & 3010 & 3488 & 6104 & 7326 & 7398 \\
\hline $2 b$ & 2614 & 2320 & 2372 & 3942 & 4092 & 2974 \\
\hline $2 c$ & 1453 & 2200 & 1906 & 3714 & 2164 & 2372 \\
\hline
\end{tabular}

hyperpolarizabilities. ${ }^{59}$ However there are some problems for systems with large charge transfer. In cases like paranitroaniline the hyperpolarizability calculations underestimate the experimental values by $16 \%$ at low frequencies and by $30 \%$ to more than $40 \%$ at higher frequencies. ${ }^{61}$ This could also explain why the molecules with larger hyperpolarizability show also larger discrepancies to experimental results.

Solvent measurements indicate a large shift in the hyperpolarizability values for charge transfer molecules ${ }^{62}$ in solutions with large dielectric constants. Allin et al. reports ratios as large as 6 between the hyperpolarizabilities calculated in vacuo and in a solvent (the average ratio is 3.7 ). ${ }^{63}$ Although solvent effects should be less important in octopolar than in linear dipolar molecules, ${ }^{37}$ the neglect of these effects adds to the previous innacuracies.

All these effects must be taken into account when comparing the calculated values with the experimental ones. Nevertheless, and since the most important trends are well reproduced, the present results allow for a detailed analysis and the drawing of interesting conclusions.

The DFT/B3LYP hyperpolarizability results obtained for the $\mathbf{D}$ series are summarized in Table 2 and plotted in Figure 4. PM3 and PM6 semiempirical results are, in general, similar and in reasonable agreement with the DFT results. There are, however, some exceptions. The geometries obtained for protonated molecules using the two semiempirical Hamiltonians and DFT/B3LYP show significant differences, and this is reflected in the hyperpolarizability values. DFT geometries and, therefore, hyperpolarizability values, are closer to PM6 values, both predicting off-plane pyridyl rings. The planar geometries obtained within the PM3 Hamiltonian for the D1 molecules lead to significantly larger hyperpolarizability values. The effect of geometry in hyperpolarizability is discussed in the next sections.

The hyperpolarizability values of the $\mathbf{D}$ series are of the same order of magnitude and display a similar pattern of
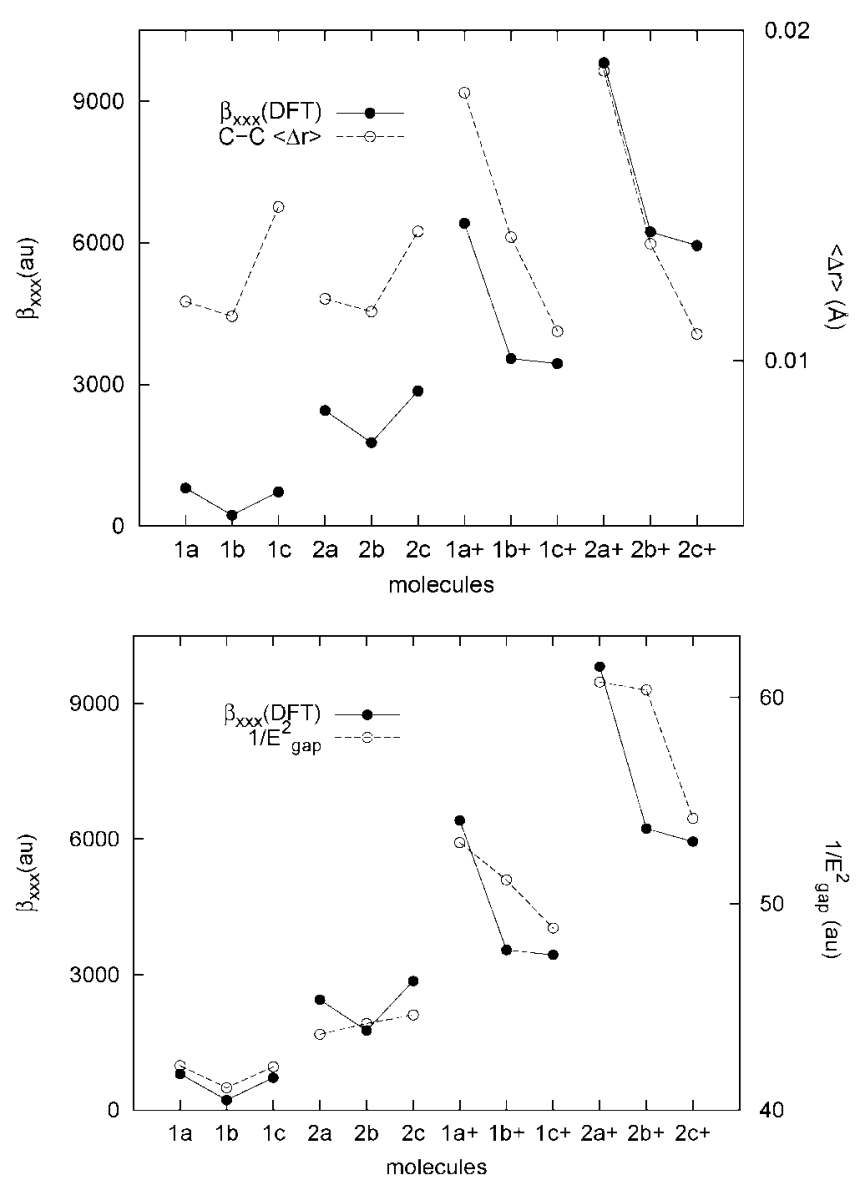

Figure 7. $\mathrm{C}-\mathrm{C}$ bond length deviation (top) and energy gaps (bottom) calculated within DFT/B3LYP for the $\mathbf{T}$ series and compared with $\beta_{x x x}$.

modulation to those of the $\mathbf{T}$ molecules (Figure 5). This is in agreement with the results reported Lee et al., in which no significant hyperpolarizability enhancement is seen when the double $\mathrm{C}-\mathrm{C}$ bond is replaced by a triple bond in a series of compounds. ${ }^{64}$ The protonated series presents larger hyperpolarizability than the neutral forms, as in the $\mathbf{T}$ molecules. However, the increase on hyperpolarizability seen from the $\mathbf{T} 1$ to the $\mathbf{T} 2$ series, due to the presence of electron rich $\mathrm{CH}_{3}$ groups which increase the donor-acceptor strength, does not happen for the D series. D2 molecules yield hyperpolarizability values similar to the $\mathbf{D} 1$ series and present lower values than its $\mathbf{T} \mathbf{2}$ counterparts. The reason for this is discussed below.

3.2. Energy Gaps. From now on we will focus on the DFT/B3LYP results. Calculations show that, for the $\mathbf{T}$ series, protonation decreases the HOMO-LUMO energy gap. There is also a decrease on the energy gap from the $\mathbf{T 1}$ to the $\mathbf{T 2}$ series. Figure 7 shows that the hyperpolarizability values follow very closely the trend of the energy gap, as predicted by the three-level model for octopolar molecules. ${ }^{65}$ The $\mathbf{D}$ series presents a more complex scenario. There is still a decrease of the gap from the neutral to the protonated series. However, for the neutral molecules, and contrarily to what happens in the $\mathbf{T}$ series, the $\mathbf{D} 2$ energy gap is larger than the D1 gap and so is its hyperpolarizability. For the charged molecules the hyperpolarizability and the energy gap of the D1 and D2 are similar but do not present a common trend. 
3.3. Molecular Geometries. 3.3.1. T Series. The molecular DFT/B3LYP geometry optimization, performed within the $C_{3}$ point group symmetry for the $\mathbf{T}$ molecules, results mainly in planar geometries. The only exception is the molecule T2a in the neutral form, which has the pyridyl rings tilted by $22^{\circ}$. This deviation does not seem to affect its hyperpolarizability, since, in the neutral series, this is the molecule that has the largest hyperpolarizability and HRS values. Also, the bonds from the central ring to the three pyridyl rings are not exactly linear, with calculations yielding bond angles that can be as large as $8^{\circ}$. There are however other differences in the molecular geometries which seem to be more important from the point of view of nonlinear optics. The most significant differences are a consequence of protonation.

We computed the elongation of the $\mathrm{C}-\mathrm{N}$ and $\mathrm{C}-\mathrm{C}$ bonds, $\left\langle R_{a v}-R_{o p t}\right\rangle,\left(R_{o p t}(N-C)=1.334 \AA, R_{o p t}(C-C)=1.388\right.$ $\AA),{ }^{66}$ using the DFT/B3LYP optimized geometries. Protonation leads to a significant elongation of the $\mathrm{C}-\mathrm{N}$ bonds in the pyridyl rings: the average value of this elongation for the neutral molecules is $0.003 \AA$ and increases to $0.020 \AA$ for the protonated molecules. This effect follows the large increase in the hyperpolarizability values.

The mean deviation of the $\mathrm{C}-\mathrm{C}$ bond lengths from their average value, $\langle\Delta r\rangle_{C-C}$, which was already identified as relevant for dipolar molecules, ${ }^{9-11}$ is superimposed with the $\mathrm{C}-\mathrm{N}$ bond elongation. Figure 7 presents a plot of $\langle\Delta r\rangle_{C-C}$ and $\left\langle\beta_{H R}\right\rangle$ values. Since the molecules in each series differ only on the $\mathrm{N}$ site in the pyridyl rings, only the $\mathrm{C}-\mathrm{C}$ bonds in the pyridyl rings and the bonds connecting the pyridyl rings to the conjugation bridge were considered. In fact, the bond length alternation in the central ring is almost negligible, with a maximum of $\langle\Delta r\rangle=0.004 \AA$. Within each series $\langle\Delta r\rangle_{C-C}$ follows very closely the hyperpolarizability values, with maxima for the protonated 1a and $\mathbf{2 a}$ molecules. It is possible that a larger $\langle\Delta r\rangle_{C-C}$ or $\mathrm{C}-\mathrm{N}$ elongation would increase further the hyperpolarizability. The ideal value determined by Marder ${ }^{67}$ for push-pull polyenes is $0.03<$ $\langle\Delta r\rangle<0.05 \AA$.

Protonation enhances the bond length alternation for molecules T1a, T1b, T2a, and T2b and has the opposite effect on molecules T1c and T2c. However, the $\mathrm{N}-\mathrm{C}$ elongation causes the hyperpolarizability of the protonated molecules to be larger even for the latter molecules. This indicates that the two structure related effects that influence the hyperpolarizability values, $\mathrm{C}-\mathrm{N}$ bond elongation due to protonation and the variation of $\langle\Delta r\rangle_{C-C}$ with the $\mathrm{N}$ site, happen mainly within the pyridyl rings.

3.3.2. D Series. In the case of the $\mathbf{D}$ series, the three methods used to optimize the molecular geometries predict different structures. In particular for the D1 series, PM3 and PM6 methods predict respectively, on plane and off-plane pyridyl rings, which leads also to different values of hyperpolarizability as will be described below. DFT/B3LYP geometries for the neutral D1 molecules are close to PM3 results. Although in the protonated case DFT/B3LYP geometries present also off-plane pyridyl rings, the deviations from planarity are smaller than $30^{\circ}$, whereas PM6 geometries
Table 3. Pyridyl-Central Benzene Dihedral Angle ${ }^{a}$

\begin{tabular}{lrrrrrrr}
\hline & \multicolumn{3}{c}{ neutral } & & \multicolumn{3}{c}{ protonated } \\
\cline { 2 - 3 } \cline { 6 - 8 } & B3LYP & PM3 & PM6 & & B3LYP & PM3 & PM6 \\
\hline 1a & -179 & 180 & -12 & & 156 & 180 & 86 \\
1b & 171 & 180 & 82 & & 131 & 180 & 83 \\
1bb & 170 & 180 & 81 & & 133 & 180 & 96 \\
1c & 179 & 180 & 166 & & 138 & 180 & 81 \\
1cc & 176 & 180 & 140 & & 132 & 180 & 61 \\
2a & 110 & 96 & 41 & & 111 & -122 & 72 \\
2b & 98 & 71 & 115 & & 100 & 126 & 102 \\
2bb & 92 & 75 & 33 & & 90 & -120 & 103 \\
2c & 117 & 125 & 137 & & 91 & -120 & 59 \\
2cc & 115 & 123 & 94 & & 93 & -77 & 44 \\
\hline & & & & & & & \\
\hline
\end{tabular}

${ }^{a}$ In degrees, computed within DFT/B3LYP, for the $\mathbf{D}$ series.

show deviations close to $90^{\circ}$. The computed dihedral angles are summarized in Table 3.

We will focus from now on only on the DFT/B3LYP results. The D1 neutral molecules are mainly planar, with pyridyl ring torsion angles below $3^{\circ}$. Upon protonation, the distortion increases to values around $50^{\circ}$. D1 is an exception, presenting an almost planar geometry. In the case of the D2 series, due to steric crowding, the $\mathrm{CH}_{3}$ group forces the pyridyl rings to be almost perpendicular to the central benzene ring, and protonation does not lead to significant changes on the torsion angles.

As the hyperpolarizability values of the $\mathbf{T}$ molecules were well correlated with structural parameters, we looked for other structural parameters that could explain the differences between the $\mathbf{T}$ and $\mathbf{D}$ series. $\mathbf{T} 1$ and $\mathbf{T} 2$ molecules are mainly planar, whereas D1 molecules are planar and D2 molecules have the pyridyl rings tilted with respect to the central benzene ring plane. This means that the stronger $\mathrm{CH}_{3}$ electron donor character of the $\mathbf{D 2}$ series, which was expected to enhance its hyperpolarizability, is somehow canceled by the rotation of the pyridyl rings due to the same $\mathrm{CH}_{3}$ groups. The relation between the deviation from planarity and the hyperpolarizability values can be seen in Figure 8 . As the dihedral angle deviates from $180^{\circ}$, the computed $\beta$ values decrease.

We compared again the hyperpolarizability values with the $\langle\Delta r\rangle$ of the pyridyl rings. For the neutral molecules, $\langle\Delta r\rangle$ shows small variations, with values between 0.007 and 0.009 $\AA$. In this case the modulation of hyperpolarizability follows very closely the variations of the dihedral angle. In the charged molecules, $\langle\Delta r\rangle$ ranges from 0.009 to $0.022 \AA$. The three properties, dihedral angle, $\langle\Delta r\rangle$, and hyperpolarizability follow the same trend, revealing the effect of the two structural parameters in the calculated hyperpolarizability values.

The calculated values suggest that, independently of the bond order present in the conjugation bridge between the donor and acceptor groups, hyperpolarizability is somehow controlled by the planarity of the molecules, the donor and acceptor strengths, and the bond length alternation. All the T molecules are planar, and therefore the hyperpolarizability values are determined by the donor and acceptor strengths (series T1 vs series T2) and, within each series, by the $\mathrm{C}-\mathrm{C}$ bond length alternation. For the $\mathbf{D}$ series, the dependence on the pyridyl dihedral angle can mask the increase of the donor-acceptor strength. 

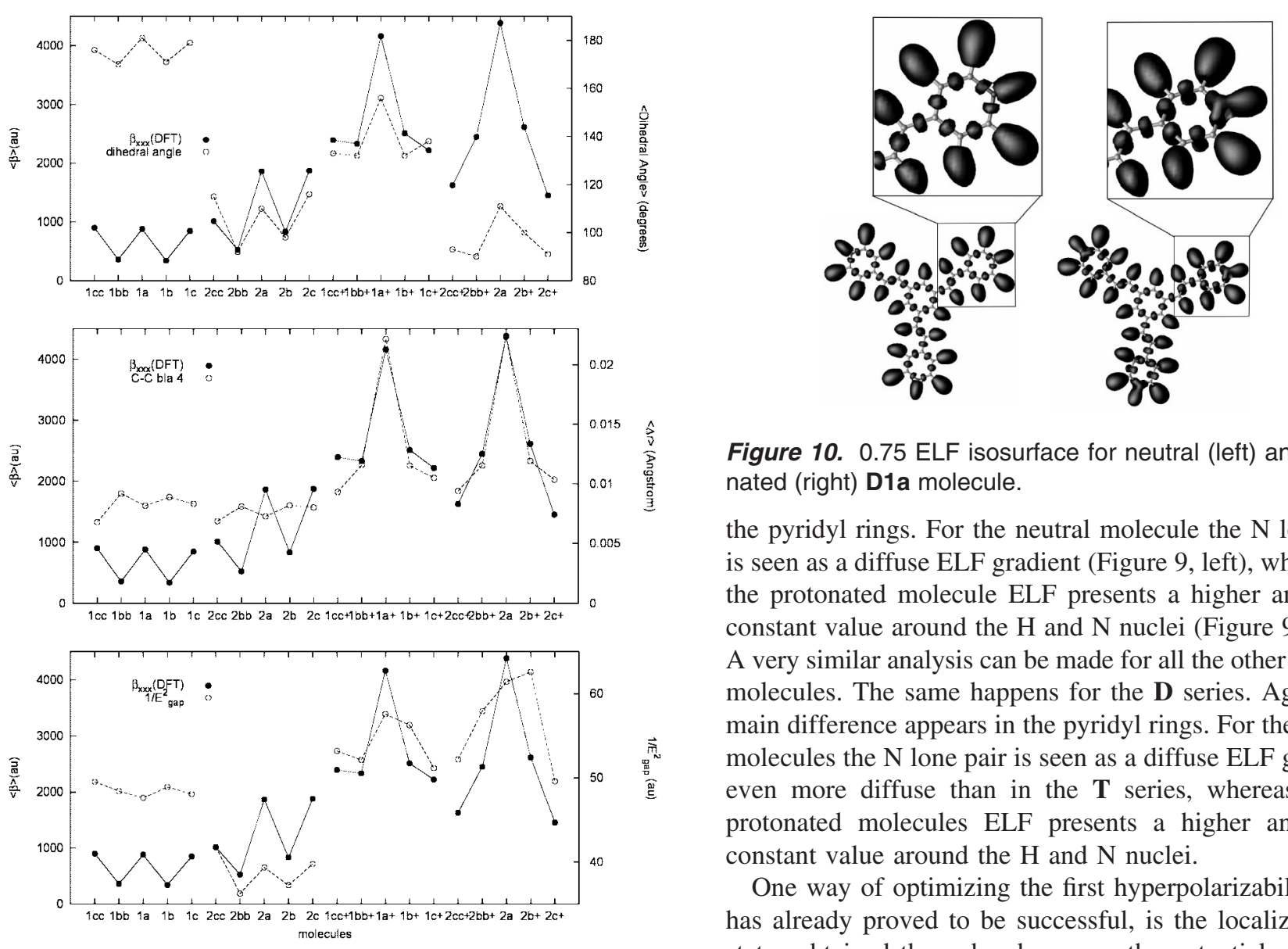

Figure 8. Torsion angles (top), $\mathrm{C}-\mathrm{C}$ bond length deviation (center), and energy gaps (bottom) calculated within DFT/ B3LYP for the D series.
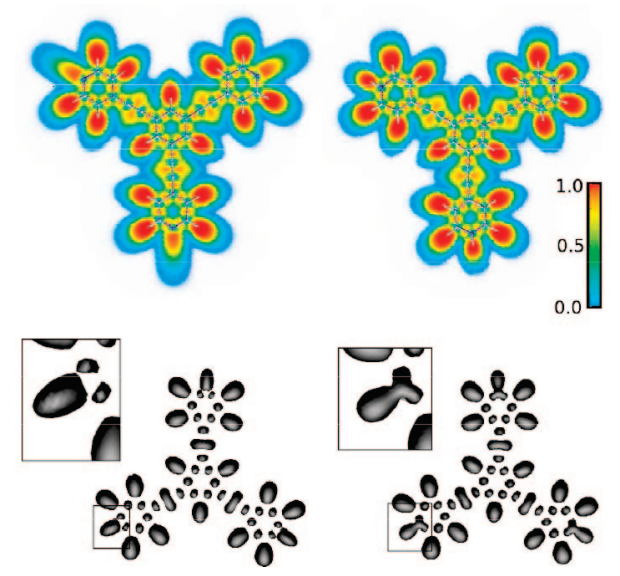

Figure 9. ELF for the $z=0$ slab (top) and $E L F=0.75$ isosurface (bottom) for neutral (left) and protonated (right) T1a molecule.

3.4. Electron Localization Function. The origin of the $\mathrm{N}-\mathrm{C}$ bond elongation is clarified by the analysis of the Electron Localization Function (ELF). Figure 9 shows the ELF, calculated for the neutral and protonated T1a molecule. In both cases there is a regular alternation between the maxima (bonds) and minima (nuclear sites, except $\mathrm{H}$ ) of the ELF along the molecular plane. The $\mathrm{C} \equiv \mathrm{C}$ triple bond in the conjugation bridge is clearly seen as a characteristic torus around the bond axis. The main difference appears in Figure 10. 0.75 ELF isosurface for neutral (left) and proto-
nated (right) D1a molecule.

the pyridyl rings. For the neutral molecule the $\mathrm{N}$ lone pair is seen as a diffuse ELF gradient (Figure 9, left), whereas in the protonated molecule ELF presents a higher and more constant value around the $\mathrm{H}$ and $\mathrm{N}$ nuclei (Figure 9, right). A very similar analysis can be made for all the other $\mathbf{T}$ series molecules. The same happens for the $\mathbf{D}$ series. Again, the main difference appears in the pyridyl rings. For the neutral molecules the $\mathrm{N}$ lone pair is seen as a diffuse ELF gradient, even more diffuse than in the $\mathbf{T}$ series, whereas in the protonated molecules ELF presents a higher and more constant value around the $\mathrm{H}$ and $\mathrm{N}$ nuclei.

One way of optimizing the first hyperpolarizability, that has already proved to be successful, is the localization of states obtained through a bump on the potential energy. ${ }^{68}$ This causes a modulation of conjugation, and the system becomes closer to the three-level model system. A similar effect can be seen in Figures 9 and 10, in which the isosurface $\mathrm{ELF}=0.75$, corresponding to a considerably high electron localization, is plotted. In the neutral molecule, the isosurface corresponding to the $\mathrm{N}$ lone pair does not reach the $\mathrm{N}$ site (Figures 9 and 10, left). The charged molecule, however, presents an isosurface that encompasses the $\mathrm{H}$ and $\mathrm{N}$ nuclei (Figures 9 and 10, right). Therefore we can conclude that protonation results in an increase of the electron localization around $\mathrm{N}$, attracting part of the electrons of the $\mathrm{N}-\mathrm{C}$ bond. As a consequence, the $\mathrm{N}-\mathrm{C}$ bond order decreases and the bond length increases, leading to a decrease of the conjugation of the pyridyl ring and a modulation of the conjugation of the system.

3.5. Wave Functions. Figure 11 shows the Kohn-Sham HOMO and LUMO orbitals for the neutral and protonated T1a molecule, computed with the Octopus code, as an example of the general behavior. The highest occupied energy levels are distributed by a set of two almost degenerate orbitals and another set of three orbitals with slightly lower energies. The neutral molecules show two unoccupied orbitals above HOMO with very similar energies, whereas the protonated molecules LUMO shows a set of three unoccupied orbitals. LUMO orbitals, both in the neutral and charged cases, extend over the whole molecule allowing the charge to travel from the donor to the acceptor fragments, a necessary condition for a large hyperpolarizability. Protonation seems to modify the HOMO orbitals. In the neutral case, three of the five highest occupied orbitals do not extend 

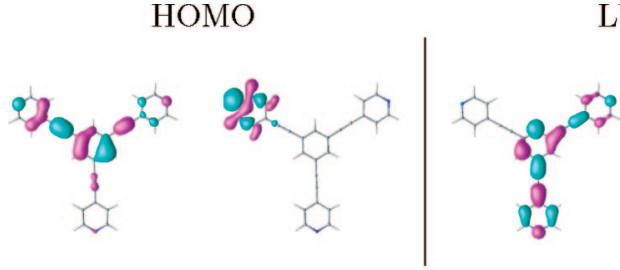

LUMO
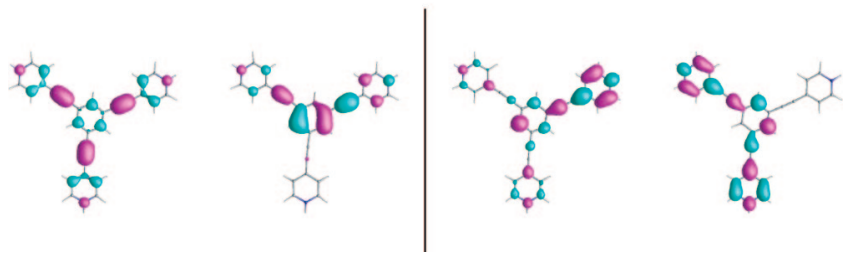

Figure 11. Kohn-Sham orbitals for neutral (top) and protonated (bottom) 1a molecules. Symmetry related degenerate orbitals are not shown.

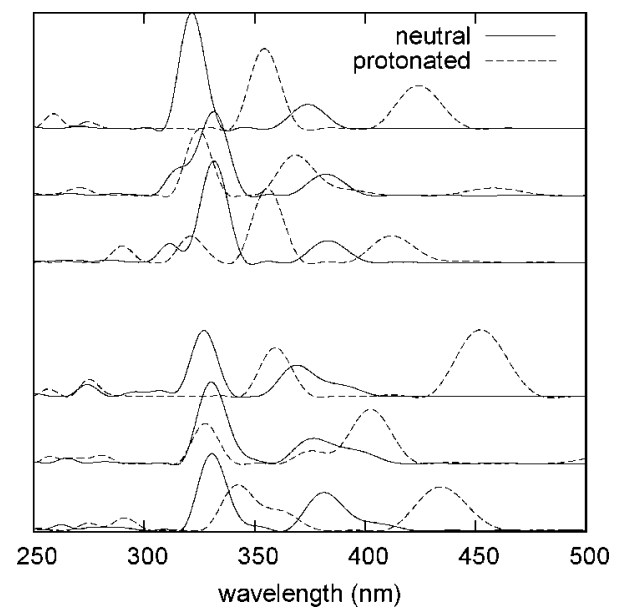

Figure 12. Calculated absorption spectra for, from top to bottom, T1a, T1b, T1c, T2a, T2b, and T2c. The solid (dashed) lines correspond to the neutral (protonated) molecules.

beyond the pyridyl rings, reducing the possibility of charge transfer, whereas in the charged case the wave functions extend over the whole molecule.

3.6. Linear Response. In Figure 12 we present the absorption spectra computed for the $\mathbf{T}$ molecules, in the neutral and charged forms (as, to our knowledge, no experimental absorption spectra exist for the $\mathbf{D}$ molecules, we will only discuss our results for the $\mathbf{T}$ series). The neutral molecules show a first peak (set of peaks in the case of T1b) between 300 and $350 \mathrm{~nm}$ and a less intense structure between 350 and $400 \mathrm{~nm}$. For molecules T1a, T1c, T2a, and T2c protonation causes a shift of the spectrum toward higher wavelengths, consistent with the decrease in $\mathrm{E}_{g a p}$. It also leads to an increase of the peak separation and a decrease of the difference in magnitude of the two major absorption peaks. The molecules T1b and T2b show a slightly different behavior. The shift due to protonation, contrary to the decrease of $\mathrm{E}_{\text {gap }}$, is small and toward lower wavelengths, indicating other transitions other than HOMO-LUMO.

For the $\mathbf{T} 2$ series there is a shoulder at the right-hand side of the peak at higher wavelength which can be explained by the energy distribution of the levels just below HOMO. For the charged molecules, there is a shift of the same peak toward higher wavelengths: from T1a to T2a, the peak at
$425 \mathrm{~nm}$ is shifted to $450 \mathrm{~nm}$; from $\mathbf{T 1} \mathbf{b}$ to $\mathbf{T 2} \mathbf{b}$ the peak at 370 goes to $400 \mathrm{~nm}$. These results are in good agreement with the experimental results which report, for the protonated forms of T1c, T2a, and T2c, broader, red-shifted absorption bands with high molar absorptivities, when compared to the neutral forms ${ }^{37,38}$ Our results show, however, peaks slightly shifted toward higher wavelengths than the experimental values, possibly due to solvent effects.

The two main peaks of the absorption spectra of the neutral molecules can be attributed to transitions between the HOMO states and the first and second sets of unoccupied states. The eigenvalue differences between HOMO and LUMO states range from 2.7 to $2.9 \mathrm{eV}$ (459-427 nm) and between HOMO and $\mathrm{LUMO}+1$ states from 3.0 to $3.4 \mathrm{eV}(413-365 \mathrm{~nm})$. This explains the splitting between the two main peaks of the absorption spectra, although it does not give their exact location. For the protonated molecules, the two main peaks correspond to transitions from the two sets of highest energy occupied states. The HOMO-LUMO eigenvalue differences range from 2.5 to $2.8 \mathrm{eV}(496-443 \mathrm{~nm})$, and the HOMO1-LUMO gap ranges from 3.1 to $3.3 \mathrm{eV}(376-400 \mathrm{~nm})$, in agreement with the red-shifted absorption spectra of the protonated molecules.

\section{Interplay between Geometric and Electronic Properties}

To date, the vast majority of effective chromophores, concerning the electro-optic activity, are planar conjugated $\pi$-electron systems with electron-donor and-acceptor moieties at their ends. As mentioned above, these chromophores exhibit a dominant intramolecular charge-transfer transition from the ground-state to the first excited-state and produce effective polarization along the $\pi$-conjugated axis. Recent results $^{69-72}$ suggest that molecules with twisted $\pi$-electron systems bridging donor (D) and acceptor (A) substituents exhibit large hyperpolarizabilities. The twist-induced reduction in D- $\pi$-A conjugation leads to aromatic stabilization and formal charge-separated zwitterionic ground states, relatively low-energy optical excitations, and large dipole moment changes from the ground state to the first excited-state.

In order to study in more detail the interplay between the molecular structures and the electronic properties we computed the $\mathbf{T}$ series hyperpolarizability for several values of the pyridyl rings torsion angle and of the $\langle\Delta r\rangle$, without a previous geometry relaxation. In Figure 13 we plot $\beta_{x x x}$ and $1 / E_{\text {gap }}^{2}$ for different torsion angles for the neutral and protonated T1a molecule. Contrarily to what happens to the chromophores reported by Kang et al. ${ }^{69}$ the increase of the torsion angle decreases significantly the hyperpolarizability: $\beta_{x x x}$ is 4 to 5.5 times larger in the case of the planar geometries than when the pyridyl rings are perpendicular to the central ring. The same happens to $1 / E_{g a p}^{2}$. However the values change in a smaller range: for the neutral molecules $1 / E_{\text {gap }}^{2}$ is 1.5 times larger for the planar geometry than for the geometry with a $90^{\circ}$ torsion angle, whereas for the protonated molecule there is no significant variation.

We also varied the $\langle\Delta r\rangle$ values for the protonated form of T1a by changing the length of the $\mathrm{C}-\mathrm{C}$ bonds in the pyridyl rings. In doing so, $\langle\Delta r\rangle$ ranges from the value of the 

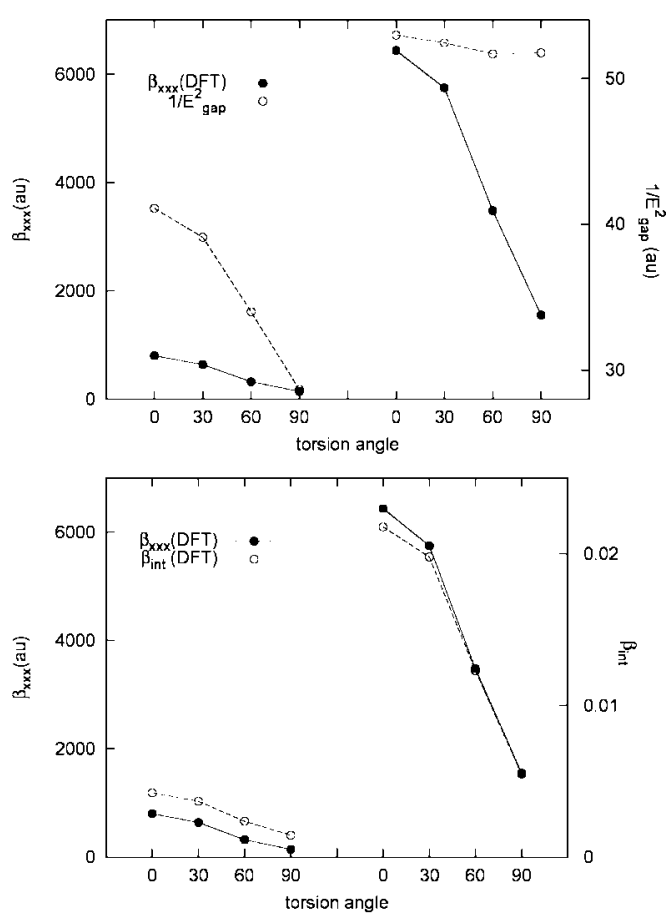

Figure 13. In the upper graph we plot $\beta_{x x x}$ and $1 / E_{\text {gap }}^{2}$ calculated for different torsion angles for the molecule T1a, both in the neutral (left) and in the protonated (right) case. In the second graph we plot the absolute and the intrinsic value of the hyperpolarizability. The intrinsic and absolute hyperpolarizability follow the same trend, as expected, since neither the size of the molecule nor the number of $\pi$ electrons change.

optimized geometry to less than half of that valuer. However, there are no significant changes in the hyperpolarizability values neither in the energy gaps.

\section{Conclusions}

$A b$ initio calculations correctly reproduce the large hyperpolarizabilities of the charged octopolar molecules, including the significant increase of first hyperpolarizability upon protonation reported in previous studies. The HOMO-LUMO energy gap decreases upon protonation, its inverse square being related to the $\beta$ values, in agreement with the threelevel model. Linear response TDDFT calculations are also in good agreement with the experimental absorption spectra, reproducing the bathochromic shift of the peaks upon protonation, due to the gap reduction mentioned above. DFT/ B3LYP calculations allowed the identification of the levels involved in the transitions.

$A b$ initio hyperpolarizability values of the protonated octopolar D molecules follow closely the values calculated for the corresponding $\mathbf{T}$ molecules, both in the neutral and protonated cases. The calculated values suggest that, independently of the bond order present in the conjugation bridge between the donor and acceptor groups, hyperpolarizability is mainly controlled by the planarity of the molecules, the donor and acceptor strengths, and the bond length alternation. All the $\mathbf{T}$ molecules are planar, and therefore the hyperpolarizability values are determined by the donor and acceptor strengths and, to a lesser degree, by the $\mathrm{C}-\mathrm{C}$ bond length alternation. For the $\mathbf{D}$ series, the dependence on the pyridyl torsion angle can mask the increase of the donor-acceptor strength.

Changes in the molecules' geometries were also analyzed, and a correlation between the molecular structures and the first hyperpolarizability, $\beta$, was suggested. Several parameters affecting $\beta$ were identified: i) the $\mathrm{C}-\mathrm{N}$ bond elongation upon protonation, which follows from the localization of the electrons around $\mathrm{H}$ and $\mathrm{N}$, as seen in the ELF plots, and accompanies the increase of the $\beta$ values; ii) the planarity of the molecules; and iii) the $\mathrm{C}-\mathrm{C}$ bond length alternation, already identified as relevant for dipolar molecules. To test these effects we computed the hyperpolarizability for several constrained geometries, thus confirming the importance of planarity on the hyperpolarizability values.

Acknowledgment. This work was supported by Fundação para a Ciência e a Tecnologia (FCT), within the project POCI/FIS/58309/2004.

\section{References}

(1) Zyss, J.; Ledoux, I. Chem. Rev. 1994, 94, 77.

(2) Nonlinear Optics of Organic Molecules and Polymers; Nalwa, H. S., Miyata, S., Eds.; CRC Press: FL, 1997.

(3) Kuzyk, M. G. SPIE Proc. 1997, 3147.

(4) Marder, S. R.; Perry, J. W.; Schaefer, P. W. Science 1989, 245, 626 .

(5) Clays, K.; Wostyn, K.; Olbrechtes, G.; Persoons, A.; Watanabe, A.; Nogi, K.; Duan, X.-M.; Okada, S.; Oikawa, H.; Nakanishi, H.; Brédas, J. L. J. Opt. Soc. Am. B 2000, 17, 256.

(6) Marder, S. R. Chem. Commun. 2006, 131.

(7) Marder, S. R.; Beratan, D. N.; Cheng, L.-T. Science 1991, 252, 103.

(8) Marder, S. R.; Gorman, C. B.; Tiemann, B. G.; Cheng, L.-T. J. Am. Chem. Soc. 1993, 115, 3006.

(9) Gorman, C. B.; Marder, S. R. Proc. Natl. Acad. Sci. U.S.A. 1993, 90, 11297.

(10) Marder, S. R.; Perry, J. W.; Tiemann, B. G.; Gorman, C. B.; Biddle, S. L.; Gilmour, S.; Bourhill, G. J. Am. Chem. Soc. 1993, 115, 2524.

(11) Bourhill, G.; Brédas, J.-L.; Cheng, L.-T.; Marder, S. R.; Meyers, F.; Perry, J. W.; Tiemann, B. G. J. Am. Chem. Soc. 1994, 116, 2619.

(12) Feringa, B. L. Molecular Switches; Wiley-VCH: Weinheim, 2001.

(13) Irie, M. Chem. Rev. 2000, 100, 1683.

(14) Coe, B. J. Chem.--Eur. J. 1999, 5, 2464.

(15) Asselberghs, I.; Clays, K.; Persoons, A.; Ward, M. D.; McCleverty, J. J. Mater. Chem. 2004, 14, 2831.

(16) Rurack, K. Spectrochim. Acta, Part A 2001, 57, 2161.

(17) Meyers, F.; Marder, S. R.; Perry, J. W. Viley-VCH: Weinheim, 1998.

(18) Verbiest, T.; Houbrechts, S.; Kauranen, M.; Clays, C.; Persoons, A. J. Mater. Chem. 1997, 7, 2175.

(19) Pond, S. J. K.; Tsutsumi, O.; Rumiand, M.; Kwon, O.; Zojerand, E.; Brédas, J. L.; Marder, S. R.; Perry, J. W. J. Am. Chem. Soc. 2004, 126, 9291. 
(20) Kim, H. M.; Jeonga, M.-Y.; Ahn, H. C.; Jeon, S.-J.; Cho, B. R. J. Org. Chem. 2004, 69, 5749.

(21) Werts, M. H. V.; Gmouh, S.; Mongin, O.; Pons, T.; BlanchardDesce, M. J. Am. Chem. Soc. 2004, 126, 16294.

(22) Asselberghs, I.; Zhao, Y.; Clays, K.; Persoons, A.; Comito, A.; Rubin, Y. Chem. Phys. Lett. 2002, 364, 279.

(23) Zyss, J. Nonlinear Opt. 1991, 1, 3.

(24) Zyss, J.; Pecaut, J.; Levy, J. P.; Masse, R. Acta Crystallogr., Sect. B: Struct. Sci. 1993, 49, 334.

(25) Ledoux, I.; Zyss, J.; Siegel, J.; Brienne, J.; Lehn, J. M. Chem. Phys. Lett. 1990, 440, 172.

(26) Ray, P. C.; Das, P. K. Chem. Phys. Lett. 1995, 244, 153.

(27) Chao, B. R.; Park, S. B.; Lee, S. J.; Son, K. H.; Lee, S. H.; Kang, T. I.; Cho, M.; Jeon, S.-J. J. Am. Chem. Soc. 2001, 123, 6421.

(28) Hennrich, G.; Asselberghs, I.; Clays, K.; Persoons, A. J. Org. Chem. 2004, 69, 5055.

(29) Traber, B.; Wolff, J. J.; Rominger, F.; Oeser, T.; Gleiter, R.; Goebel, M.; Wortmann, R. Chem.--Eur. J. 2004, 10, 1227.

(30) Stadler, S.; Brauchle, Ch.; Brandl, S.; Gompper, R. Chem. Mater. 1996, 8, 676.

(31) Luo, Y.; Cesar, A.; Agren, H. Chem. Phys. Lett. 1996, 252, 389.

(32) Zhu, W.; Wu, G.-S. J. Phys. Chem. A 2001, 105, 9568.

(33) Dhenaut, C.; Ledoux, I.; Samuel, I. D. W.; Zyss, J.; Bourgault, M.; Bozec, H. L. Nature 1995, 374, 339.

(34) Viau, L.; Bidault, S.; Maury, O.; Brasselet, S.; Ledoux, I.; Zyss, J.; Ishow, E.; Nakatani, K.; Le Bozec, H. J. Am. Chem. Soc. 2004, 126, 8386.

(35) Sporer, C.; Marcen, S.; Montant, S.; Létard, J. F.; Freysz, E.; Rovira, C.; Ratera, I.; Ruiz-Molina, D.; Veciana, J. Polyhedron 2003, 22, 1851.

(36) Cifuentes, M. P.; Humphrey, M. G. J. Organomet. Chem. 2004, 689, 3968.

(37) Asselberghs, I.; Hennrich, G.; Clays, K. J. Phys. Chem. A 2006, $110,6271$.

(38) Oliva, M. M.; Casado, J.; Navarrete, J. T. L.; Hennrich, G.; Delgado, M. C. R. J. Phys. Chem. C 2007, 111, 18778.

(39) Ray, P. C.; Leszczynski, J. Chem. Phys. Lett. 2004, 399, 162.

(40) Ray, P. C.; Leszczynski, J. J. Phys. Chem. A 2005, 109, 6689.

(41) Ray, P. C. Chem. Phys. Lett. 2004, 394, 354.

(42) Ray, P. C. Chem. Phys. Lett. 2004, 395, 269.

(43) Becke, A. D. J. Chem. Phys. 1993, 98, 5648.

(44) Stephens, P. J.; Devlin, F. J.; Chablowski, C. F.; Frisch, M. J. J. Phys. Chem. 1994, 98, 11623.

(45) Schmidt, M. W.; Baldridge, K. K.; Boatz, J. A.; Elbert, S. T.; Gordon, M. S.; Jensen, J. H.; Koseki, S.; Matsunaga, N.; Nguyen, K. A.; Su, S.; Windus, T. L.; Dupuis, M.; Montgomery, J. A., Jr. J. Comput. Chem. 1993, 14, 1347.

(46) Krishnan, R.; Binkley, J. S.; Seeger, R.; Pople, J. A. J. Chem. Phys. 1980, 72, 650.
(47) Castro, A.; Appel, H.; Oliveira, M.; Rozzi, C. A.; Andrade, X.; Lorenzen, F.; Marques, M. A. L.; Gross, E. K. U.; Rubio, A. Phys. Status Solidi B 2006, 243, 2465.

(48) Marques, M. A. L.; Castro, A.; Bertsch, G. F.; Rubio, A. Comput. Phys. Commun. 2003, 151, 60.

(49) Becke, A. D.; Edgecombe, K. E. J. Chem. Phys. 1990, 92, 5397.

(50) Stewart, J. J. P. Stewart Computational Chemistry; Colorado Springs, CO, U.S.A. http://openmopac.net (accessed Feb 2007).

(51) Stewart, J. J. P. J. Comput. Chem. 1989, 10, 209.

(52) Stewart, J. J. P. J. Comput. Chem. 1989, 10, 221.

(53) Stewart, J. J. P. J. Comput. Chem. 1991, 12, 320.

(54) Stewart, J. J. P. J. Mol. Model. 2004, 10, 155.

(55) Stewart, J. J. P. J. Mol. Model. 2007, 13, 1173.

(56) Zhou, J.; Kuzyk, M. G.; Watkins, D. S. Opt. Lett. 2006, 31, 2891.

(57) Benkova, Z.; Sadlej, A. J.; Oakes, R. E.; Bell, S. E. J. J. Comput. Chem. 2005, 26, 145.

(58) Benkova, Z.; Sadlej, A. J.; Oakes, R. E.; Bell, S. E. J. Theor. Chem. Acc. 2005, 113, 238.

(59) Sałek, P.; Helgaker, T.; Vahtras, O.; Grenz, H. A.; Jonsson, D.; Gauss, J. Mol. Phys. 2005, 103, 439.

(60) Cohen, A. J.; Handy, N. C.; Tozer, D. J. Chem. Phys. Lett. 1999, 303, 391.

(61) Sałek, P.; Vahtras, O.; Helgaker, T.; Ågren, H. J. Chem. Phys. 2002, 117, 9630.

(62) Teng, C. C.; Garito, A. F. Phys. Rev. B 1983, 28, 6766.

(63) Allin, S. B.; Leslie, T. M.; Lumpkin, R. S. Chem. Mater. 1996, $8,428$.

(64) Lee, S. H.; Park, J. R.; Jeong, M.-Y.; Kim, H. M.; Li, S.; Song, J.; Ham, S.; Jeon, S.-J.; Cho, B. R. Chem. Phys. Chem 2006, 7, 206.

(65) Joffre, M.; Yaron, D.; Silbey, R. J.; Zyss, J. J. Chem. Phys. 1992, 97, 5607.

(66) Krygowski, T.; Cyrański, M. Tetrahedron 1995, 52, 10225.

(67) Marder, S. R.; Gorman, C. B.; Meyers, F.; Perry, J. W.; Bourhill, G.; Brédas, J.-L.; Pierce, B. M. Science 1994, 265, 632.

(68) Pérez-Moreno, J.; Zhao, Y.; Clays, K.; Kuzyk, M. G. Opt. Lett. 2007, 32, 59.

(69) Kang, H.; Facchetti, A.; Jiang, H.; Cariati, E.; Righetto, S.; Ugo, R.; Zuccaccia, C.; Macchioni, A.; Stern, C. L.; Liu, Z.; Ho, S.-T.; Brown, E. C.; Ratner, M. A.; Marks, T. J. J. Am. Chem. Soc. 2007, 129 (11), 3267-3286.

(70) Keinan, S.; Zojer, E.; Brédas, J.-L.; Ratner, M. A.; Marks, T. J. THEOCHEM 2003, 633, 227.

(71) Albert, I. D. L.; Marks, T. J.; Ratner, M. A. J. Am. Chem. Soc. 1998, 120, 11174.

(72) Albert, I. D. L.; Marks, T. J.; Ratner, M. A. J. Am. Chem. Soc. 1997, 119, 3155.

CT800464T 